\title{
Histogram analysis of computed tomography numbers of clinical T1 NO MO lung adenocarcinoma, with special reference to lymph node metastasis and tumor invasiveness
}

\author{
Hiroaki Nomori, MD, PhD \\ Takashi Ohtsuka, MD \\ Tsuguo Naruke, MD, PhD \\ Keiichi Suemasu, MD, PhD
}

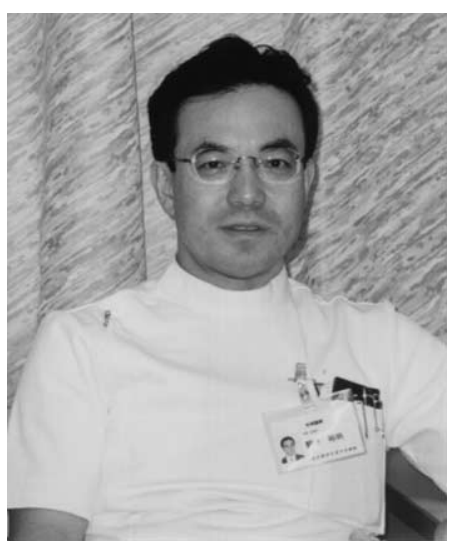

Dr Nomori

From the Department of Thoracic Surgery, Saiseikai Central Hospital, Tokyo, Japan.

Received for publication Jan 9, 2003; revisions requested April 1, 2003; revisions received April 24, 2003; accepted for publication June 3, 2003.

Address for reprints: Hiroaki Nomori, MD, Department of Thoracic Surgery, Saiseikai Central Hospital, 1-4-17 Mita, Minato-ku, Tokyo 108-0073, Japan (E-mail: hnomori@qk9.so-net.ne.jp).

J Thorac Cardiovasc Surg 2003;126:1584-9

Copyright $\odot 2003$ by The American Association for Thoracic Surgery

$0022-5223 / 2003 \$ 30.00+0$

doi:10.1016/S0022-5223(03)00885-7
Purpose: To predict lymph node metastasis and tumor invasiveness in lung adenocarcinoma from computed tomography findings, we examined computed tomography number histograms of clinical T1 N0 M0 lung adenocarcinomas.

Patients and Methods: Histograms of pixel computed tomography numbers were made for 100 patients with clinical T1 N0 M0 lung adenocarcinoma. Pathological tumor stages were $\mathrm{N} 0$ in 80 patients, $\mathrm{N} 1$ in $7, \mathrm{~N} 2$ in 9 , and T4 due to intrapulmonary metastasis in 4 .

Results: The histogram showed 3 patterns: 1 peak at a low computed tomography number $(\mathrm{n}=18), 1$ peak at a high computed tomography number $(\mathrm{n}=54)$, and 2 peaks at both low and high computed tomography numbers $(n=28)$. Histologically, adenocarcinoma with 1 peak at a low computed tomography number showed a large area of bronchioloalveolar carcinoma-like spread with little area of solid growing tumor or central fibrosis, whereas those with 1 peak at a high computed tomography number showed a large area of solid growing tumor or central fibrosis with little bronchioloalveolar carcinoma-like spread. Adenocarcinomas with 2 peaks had both types of areas. Lymph node or pulmonary metastases were seen in none $(0 \%)$ of the adenocarcinomas with 1 peak at a low computed tomography number, in $1(4 \%)$ with 2 peaks, and in $20(37 \%)$ with 1 peak at a high computed tomography number. The former 2 types had metastases less frequently than those with 1 peak at a high computed tomography number $(P<.01)$. In the 79 patients with pathological T1 N0 M0, tumor involvement of the intratumoral vessels or pleura was seen in 1 of 18 (6\%) adenocarcinomas with 1 peak at a low computed tomography number, which was significantly less frequent than the 18 of $34(53 \%)$ with 1 peak at a high computed tomography number $(P<.001)$ and 10 of $27(37 \%)$ with 2 peaks $(P<.05)$.

Conclusion: Clinical T1 N0 M0 adenocarcinomas with 1 peak at a low computed tomography number on histogram seldom had lymph node metastasis or tumor involvement of vessels or pleura. Limited surgical resection could be indicated for this type of adenocarcinoma, especially for elderly patients or patients with poor pulmonary function.

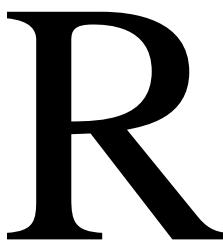

ecent advances in low-dose helical computed tomography (CT) and video-assisted thoracoscopic surgery have enabled the diagnosis of small-sized lung cancers. ${ }^{1-6}$ Although limited resection procedures, such as lung wedge resection or segmentectomy, can cure some clinical T1 N0 M0 non-small cell lung cancers, ${ }^{7-9}$ lymph node metastases are still found in approximately $20 \%$ of clinical T1 N0 M0 lung adenocarcinomas. ${ }^{10-14}$ To predict which T1 N0 M0 tumors are curable by limited resection from CT findings, several authors have evaluated the importance of ground-glass opacity (GGO) within tumors, which usually show bronchioloalveolar carcinoma (BAC)-like spread, and have shown that adenocarcinomas with large areas of GGO are more frequently of N0 stage than those with small GGO area. ${ }^{9,15-19}$ 

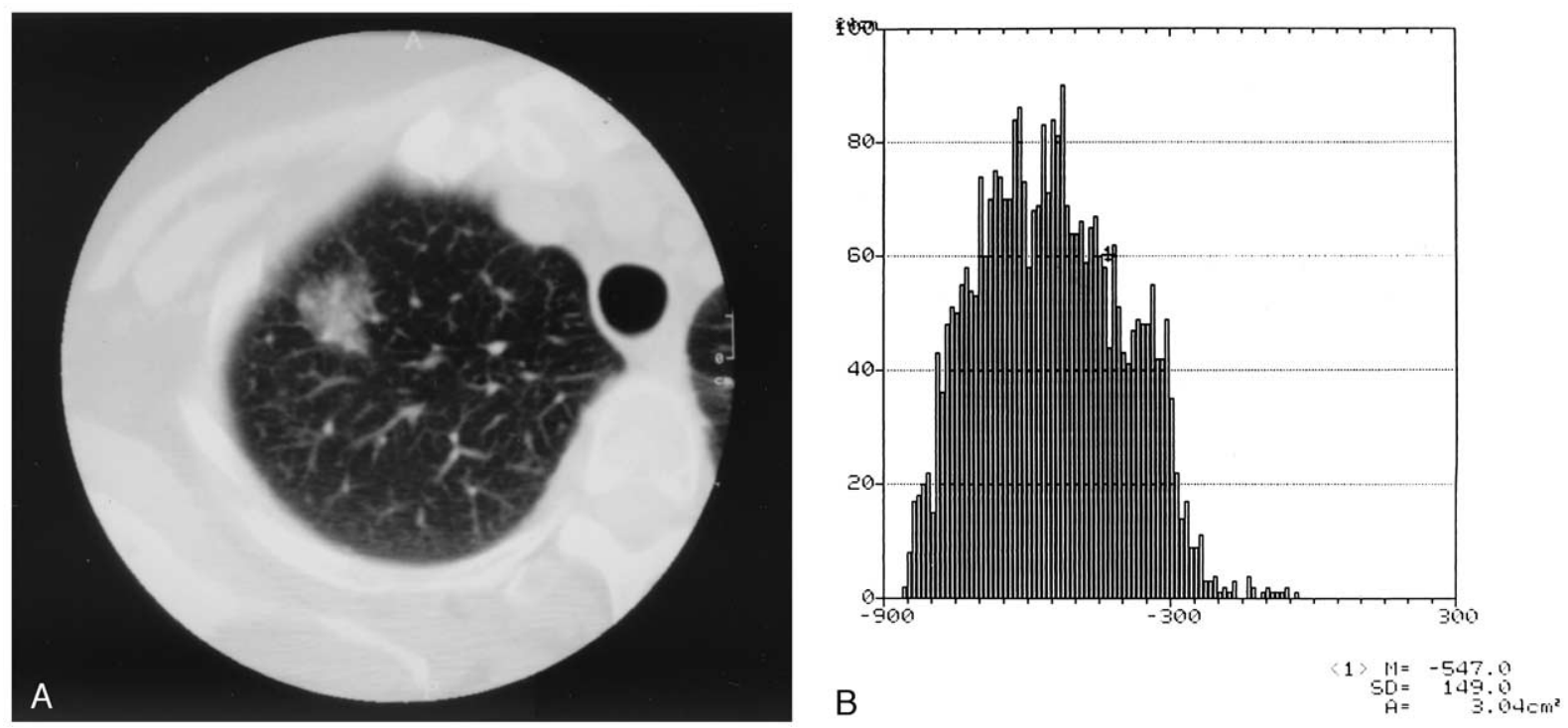

Figure 1. Adenocarcinoma with 1 peak at a low CT number on the histogram. A, CT showed a large area with low CT number in the tumor. Pathological stage was T1 NO MO. B, CT number histogram had a peak at $-500 \mathrm{HU}$.

Although the term "GGO" is used to describe a hazy increased attenuation of the lung on $\mathrm{CT}$ with preservation of the bronchial and vascular margins, the criteria for defining GGO are as "hazy" as its image on CT scans. Several authors have classified adenocarcinomas with the ratio of GGO area, ${ }^{15-19}$ but the differentiation is also kind of subjective. To perform an objective evaluation of the CT findings in small lung adenocarcinoma for predicting lymph node metastasis and tumor invasiveness, we examined CT number histograms.

\section{Materials and Methods}

Histological criteria were based on the 1999 World Health Organization histologic classification. ${ }^{20,21}$ BAC was classified from usual adenocarcinoma (ie, a tumor exhibiting a pure bronchioloalveolar growth pattern with an increase in thickness of the alveolar septa and no evidence of stromal, vascular, or pleural invasion). Between January 1997 and November 2002, 303 patients with primary lung cancer underwent surgical resection at our hospital. One hundred fifty-nine were diagnosed as having clinical T1 N0 M0 lung cancer, of whom 110 patients had adenocarcinoma. Of the 110 patients with adenocarcinoma, 105 patients underwent major lung resection and complete mediastinal lymph node dissection. We excluded 3 patients with double lung adenocarcinomas. We also excluded 2 patients with mucinous BAC because their CT numbers were strongly affected by the presence of mucin within the tumor but not by the tumor growth pattern itself. As a result, we studied 100 patients with clinical T1 N0 M0 adenocarcinoma. The medical record of each patient was examined with regard to age, gender, maximum tumor diameter, serum level of carcinoembryonic antigen (CEA) $(<5 \mathrm{ng} / \mathrm{mL}$ versus $\geq 5 \mathrm{ng} / \mathrm{mL})$, operative procedure, pathologic TNM stage, pleural involvement (p0 versus $\mathrm{p} 1$ to $\mathrm{p} 3$ ), tumor invasion of the intratumoral vessels (ie, vascular or lymphatic invasion; positive versus negative), and survival data. To examine tumor involvement of the pleura or intratumoral vessels, we routinely conducted elastica van Gieson staining. Pleural involvement was classified as p0, p1, p2, or p3: the $\mathrm{p} 0$ tumor did not extend beyond the elastic pleural layer; the $\mathrm{p} 1$ tumor invaded the visceral pleural elastic layer but did not expose itself on the pleural surface; the p2 included tumor exposed on the pleural surface; and the p3 tumor invaded the parietal pleura or chest wall. The stage of diseases was based on the TNM classification of the Internal Union Against $\mathrm{Cancer}^{22}$ : tumors with p2 were classified as T2; tumors with p3 as T3; and tumors that had intrapulmonary metastasis within the same lobe as T4. All of the patients were followed up after surgery at 3- to 6-month intervals.

\section{Histogram of CT Numbers}

The following acquisition parameters were used on the CT scanner (ProSeed SA; General Electric Medical System, Milwaukee, Wis): high voltage $(120 \mathrm{kV})$, tube load of $160 \mathrm{~mA}$, window level of -500 Hounsfield units (HU), window width of $1500 \mathrm{HU}$, and a $512 \times 512$ matrix corresponding to a pixel size of about $0.6 \mathrm{~mm}$. Air calibration was conducted every morning before operation of the scanner. The lesions less than $1.5 \mathrm{~cm}$ were subjected to scanning with 1 - to 3 -mm-thick sections at 1 breath hold with maximum inspiration, and the larger lesions were scanned with 3to 5-mm-thick sections. Therefore, each tumor was scanned with at least 4 slices. Each slice at the end of tumors was excluded, and then the slice of tumor with the densest area was selected because it should include the most malignant component of tumors. The circumference of the tumor was traced on the screen. A histogram was created from the CT numbers of each pixel within the tumor. The CT number at the peak in the histogram was quantified. 

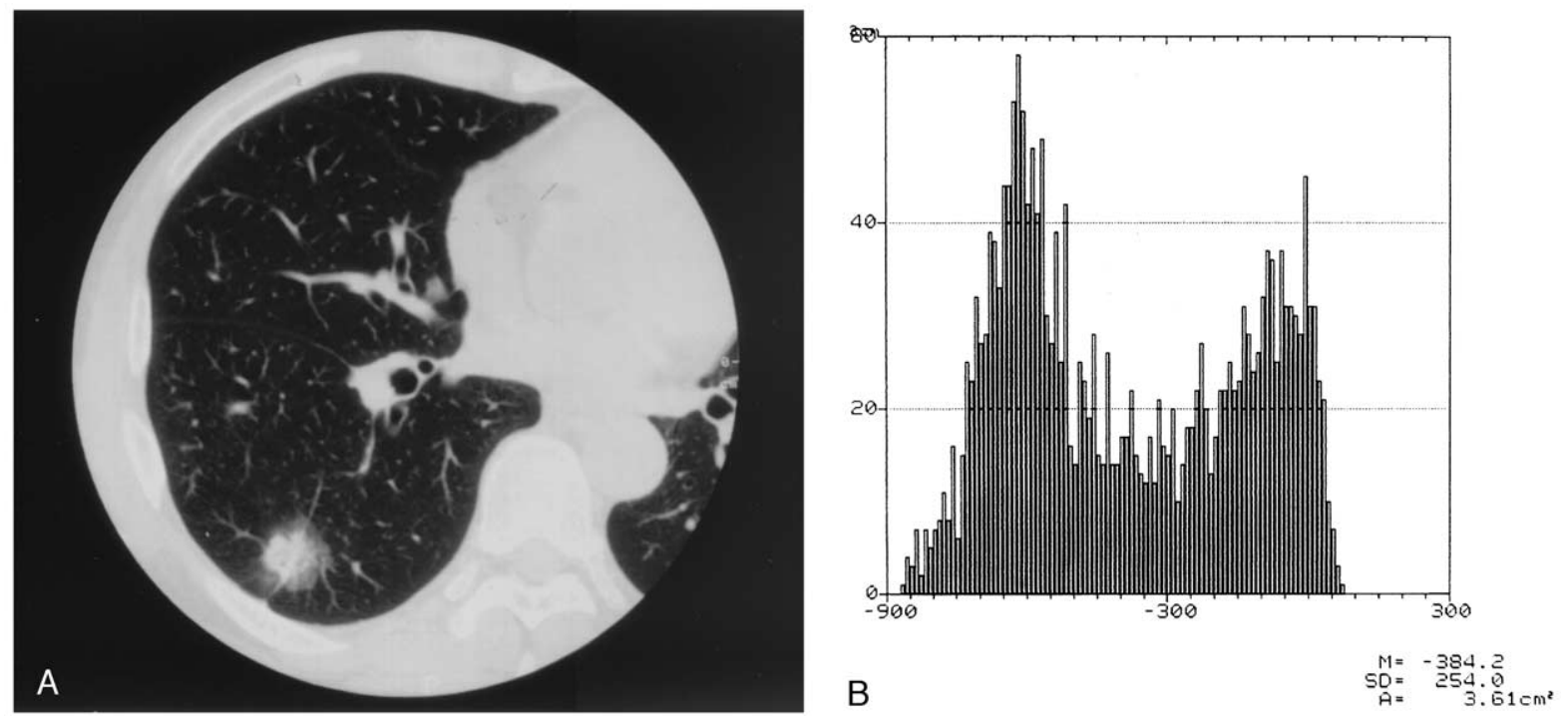

Figure 2. Adenocarcinoma with 2 peaks on the CT number histogram. A, CT showed an area with low CT number at the periphery and an area with high CT number at the center of the tumor. Pathological stage was T1 NO MO. B, CT number histogram had 2 peaks at -600 and $0 \mathrm{HU}$.

All data were analyzed for significance by using the 2-tailed Student $t$ test. Values of $P<.05$ were accepted as significant. All values in the text and tables are given as mean $\pm \mathrm{SD}$.

\section{Results}

All of the tumors were peripheral-type lung adenocarcinoma. Mean tumor size on CT image was $1.8 \pm 0.7 \mathrm{~cm}$. There were 62 men and 38 women. Their mean age was 63 \pm 11 years. The operative procedures were lobectomy in 97 patients and segmentectomy in 3. The CT number histogram showed 3 patterns (Figures 1, 2, and 3): 1 with 1 peak at a low CT number (mean: $-448 \pm 152 \mathrm{HU}$, range: -680 to $-240 \mathrm{HU}, \mathrm{n}=18), 1$ with 1 peak at a high $\mathrm{CT}$ number (mean: $23 \pm 39 \mathrm{HU}$, range: -100 to $80 \mathrm{HU}, \mathrm{n}=54$ ), and 1 with 2 peaks at both the low and high CT number zones $(n=28)$. The vertical line in each histogram shows the number of pixels within the tumor. The horizontal axis shows the CT numbers, for each of which the class interval was $10 \mathrm{HU}$ and the range -900 to $300 \mathrm{HU}$.

Histologically, adenocarcinomas with 1 peak at a low CT number showed a large area of BAC-like spread but usually little area of solid growing tumor or central fibrosis. Adenocarcinomas with 1 peak at a high CT number showed a large area of solid growing tumor or central fibrosis with little BAC-like spread. Adenocarcinomas with 2 peaks had both areas of BAC-like spread at the periphery of the tumor and solid growing tumor or central fibrosis. Ten of the 18 adenocarcinomas with 1 peak at a low CT number were classified as BAC, while none of adenocarcinomas with 1 peak at a high CT number or 2 peaks were BAC.

Table 1 shows the patient characteristics, mean tumor size, serum level of CEA, and tumor location for each histogram type. There was no significant difference in mean age and sex ratio among these 3 types. Adenocarcinomas with 1 peak at a low CT number were significantly smaller than those with 1 peak at a high CT number $(P<.05)$ but there was no significant difference between adenocarcinomas with 1 peak at a low CT number and those with 2 peaks. None of the patients who had adenocarcinomas with 1 peak at a low CT number $(0 \%)$ had high serum levels of CEA; this was significantly less frequent than the occurrence of high serum CEA in the 20 patients (37\%) with adenocarcinomas with 1 peak at a high CT number and the $9(32 \%)$ with 2 peaks $(P<.01)$. There was no significant difference in tumor location among these 3 types.

Table 2 shows the correlations between histogram pattern and tumor stage. All adenocarcinomas (100\%) with 1 peak at a low CT number were T1 N0 M0. Tumors with 1 peak at a high $\mathrm{CT}$ number were $\mathrm{N} 0$ stage in 34 patients (63\%), N1 in 7, N2 in 9, and T4 due to intrapulmonary metastasis in 4 . Tumors with 2 peaks were N0 stage in 27 patients (96\%) and N2 in 1. Adenocarcinomas with 1 peak at a low CT number and those with 2 peaks were more frequently pathological T1 N0 M0 stage than those with 1 peak at a high CT number $(P<.01)$.

Table 3 shows the correlations between histogram pattern and tumor involvement of the intratumoral vessels or pleura in the 79 patients with pathological T1 N0 M0. Of the 18 patients with 1 peak at a low CT number, 1 patient (6\%) had involvement of the lymphatic vessels, whereas the other 17 had no tumor involvement of the vessels or the pleura. Of the 34 patients with 1 peak at a high CT number, 18 $(53 \%)$ had involvement of the vessels or pleura, whereas 

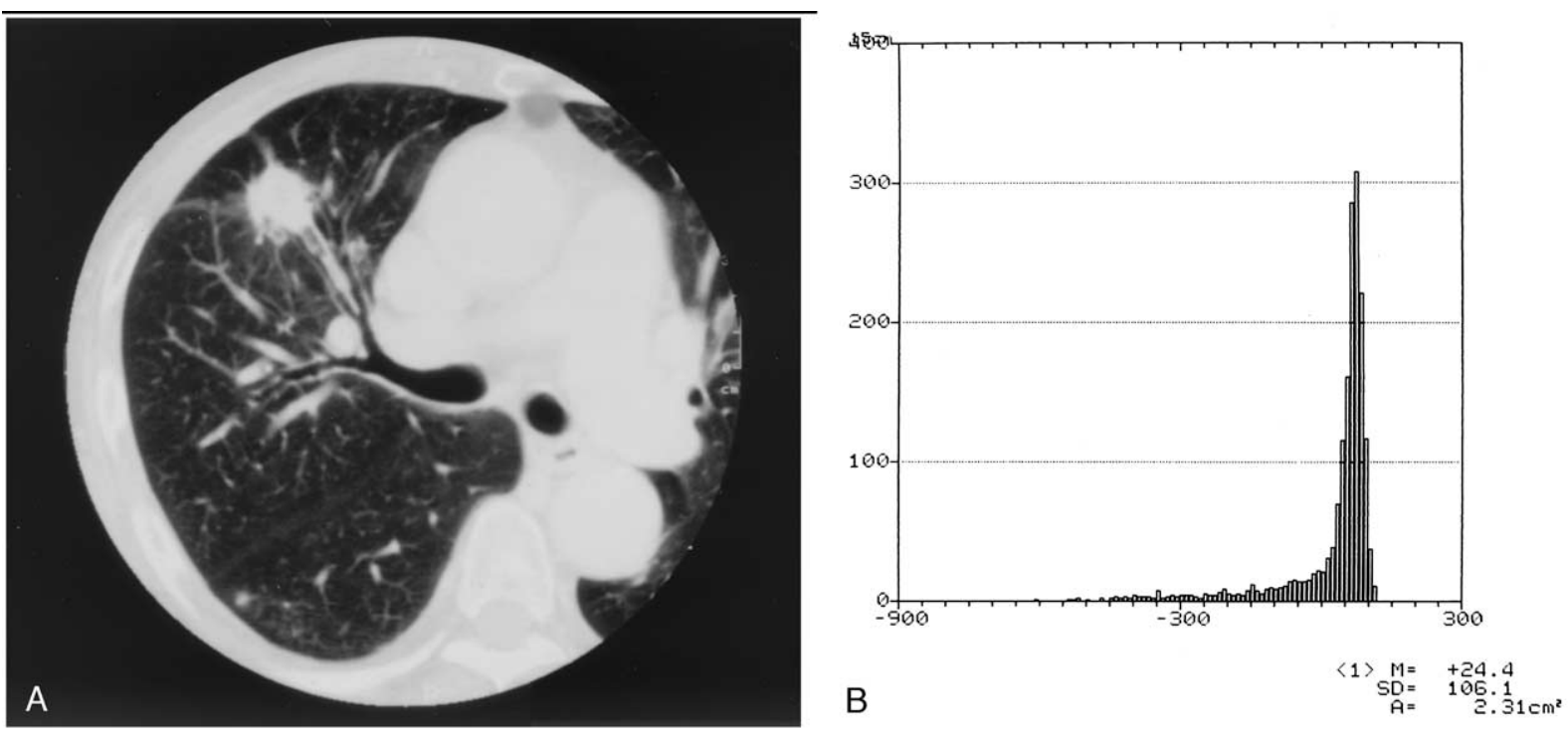

Figure 3. Adenocarcinoma with 1 peak at high CT number on histogram. A, CT showed a large area with high CT number with little area with low CT number. Pathological stage was T4 N1 MO. B, CT number histogram had a peak at $50 \mathrm{HU}$.

TABLE 1. Tumor size, serum level of carcinoembryonic antigen, and patient characteristics in each histogram pattern

\begin{tabular}{lccc}
\hline & \multicolumn{3}{c}{ Histogram pattern } \\
\cline { 2 - 4 } & $\begin{array}{c}\text { One peak } \\
\text { (low) }\end{array}$ & $\begin{array}{c}\text { One peak } \\
\text { (high) }\end{array}$ & $\begin{array}{c}\text { Two } \\
\text { peaks }\end{array}$ \\
\hline Age (y) & $61 \pm 7$ & $62 \pm 11$ & $65 \pm 11$ \\
Gender & & & \\
$\quad$ Male & 10 & 35 & 17 \\
Female & 8 & 19 & 11 \\
Tumor size (cm) & $1.4 \pm 0.6$ & $1.9 \pm 0.7$ & $1.8 \pm 0.7$ \\
CEA (ng/mL) & 18 & 34 & 19 \\
$\quad<5.0$ & 0 & 20 & 9 \\
$\geq 5.0$ & 6 & 16 & 15 \\
Tumor location & 2 & 5 & 0 \\
$\quad$ Right upper lobe & 4 & 12 & 8 \\
Right middle lobe & 3 & 16 & 5 \\
Right lower lobe & 3 & 5 & 0 \\
Left upper lobe & 18 & 54 & 28 \\
$\quad$ Left lower lobe & & & \\
Total & & & \\
\hline
\end{tabular}

One peak (low), 1 peak at low CT number; one peak (high), 1 peak at high CT number; $C E A$; carcinoembryonic antigen.

the other 16 patients had no involvement. Of the 27 patients with 2 peaks, $10(37 \%)$ had involvement of the vessels or pleura, whereas the other 17 had no involvement. Tumor involvement of the vessels or pleura was thus seen less frequently in patients with 1 peak at a low CT number than in those with 1 peak at a high CT number and those with 2 peaks $(P<.001$ and $P<.05$, respectively).
TABLE 2. Correlation between tumor stage and histogram pattern

\begin{tabular}{lcccr}
\hline & \multicolumn{3}{c}{ Histogram pattern } & \\
\cline { 2 - 4 } Tumor stage & $\begin{array}{c}\text { One peak } \\
\text { (low) }\end{array}$ & $\begin{array}{c}\text { One peak } \\
\text { (high) }\end{array}$ & $\begin{array}{c}\text { Two } \\
\text { peaks }\end{array}$ & Total \\
\hline T1 N0 M0 & 18 & 34 & 27 & 80 \\
T1 N1 M0 & 0 & 4 & 0 & 4 \\
T2 N1 M0 & 0 & 3 & 0 & 3 \\
T1 N2 M0 & 0 & 7 & 1 & 7 \\
T2 N2 M0 & 0 & 1 & 0 & 1 \\
T3 N2 M0 & 0 & 1 & 0 & 1 \\
T4 N0 M0 & 0 & 3 & 0 & 3 \\
T4 N1 M0 & 0 & 1 & 0 & 1 \\
Total & 18 & 54 & 28 & 100 \\
\hline
\end{tabular}

One peak (low); one peak at low CT number; one peak (high); one peak at high CT number.

$\mathrm{T} 2$ is classified from pleural involvement grade, p2; $\mathrm{T} 3$ is classified from pleural involvement grade, p3; T4 is classified from intrapulmonary metastasis.

Follow-up period after surgery ranged from 5 to 63 months, of which mean value was $33 \pm 13$ months. Regarding survival in the 79 patients with pathological T1 N0 M0, 4 patients with 1 peak at a high CT number and 2 patients with 2 peaks died of their tumors, whereas all patients with 1 peak at a low CT number are still alive without recurrence.

\section{Discussion}

We examined the peak CT number on histogram rather than the mean CT number for the following reasons: (1) although 
TABLE 3. Correlation between tumor involvements into intratumoral vessels or pleura and histogram pattern in adenocarcinomas with pathological T1 NO MO

\begin{tabular}{|c|c|c|c|c|c|}
\hline \multicolumn{2}{|c|}{$\begin{array}{l}\text { Tumor } \\
\text { involvement } \\
\end{array}$} & \multicolumn{3}{|c|}{ Histogram pattern } & \multirow[b]{2}{*}{ Total } \\
\hline Vessels & Pleura & One peak (low) & One peak (high) & Two peaks & \\
\hline- & - & 17 & 16 & 17 & 50 \\
\hline+ & - & 1 & 10 & 8 & 19 \\
\hline- & + & 0 & 3 & 0 & 3 \\
\hline+ & + & 0 & 5 & 2 & 7 \\
\hline Total & & 18 & 34 & 27 & 79 \\
\hline
\end{tabular}

One peak (low), one peak at low CT number; one peak (high); one peak at high CT number.

the mean CT number is easy to calculate, it can be affected by the densities of vessels or bronchi within the tumor, especially in GGO lesions; (2) the peak CT number on the histogram, on the other hand, is the most frequent value of the tumor, and by using it we can ignore the effect of vessels and bronchi within the tumor; and (3) adenocarcinomas with 2 peaks of CT number can be represented by CT histograms but not by mean $\mathrm{CT}$ number.

We classified the histograms into 3 patterns: 1 peak at a high CT number, 1 peak at a low CT number, and 2 peaks. We classified the peaks of CT number histogram into 2 zones because there was a significant gap between the low zone $(-680$ to $-240 \mathrm{HU})$ and the high zone $(-100$ to 80 $\mathrm{HU}$ ); that is, none of adenocarcinomas had peaks between -240 and $-100 \mathrm{HU}$. As a result, adenocarcinomas with 1 peak at a low CT number usually showed a large area of BAC-like spread with little area of solid tumor growth or central fibrosis. On the other hand, adenocarcinomas with 1 peak at a high CT number usually showed a large area of solid tumor growth or central fibrosis with little area of BAC-like spread, as in moderately or poorly differentiated adenocarcinomas. It has been reported that well-differentiated adenocarcinomas are more frequently of N0 stage than moderately or poorly differentiated ones. ${ }^{10,23}$ Several authors have also reported that an increased amount of central fibrosis in well-differentiated adenocarcinoma increases the frequency of lymph node metastasis. ${ }^{10,23-26}$ These previous studies support our results, which showed that adenocarcinomas with 1 peak at a low CT number had less frequent lymph node metastasis and tumor involvement of the vessels or pleura than those with 1 peak at a high CT number or with 2 peaks.

Several authors have classified the CT findings of small adenocarcinoma with the ratio of GGO area. ${ }^{15-19}$ These authors reported that lesions with larger areas of GGO were histologically BAC or well-differentiated adenocarcinoma with little fibrosis, more frequent N0, and had a better prognosis than those with smaller GGO areas. The 18 ade- nocarcinomas with 1 peak at a low CT number in this study included 10 BACs and 8 well-differentiated adenocarcinomas with little fibrosis. Although BAC is well known to be an in situ lung adenocarcinoma, ${ }^{23}$ differentiating between BAC and well-differentiated adenocarcinoma with little fibrosis is sometimes difficult. On the other hand, welldifferentiated adenocarcinomas with little fibrosis usually do not metastasize to the lymph nodes, which is why it is also referred to as "minimally invasive adenocarcinoma.", 25 Although the criteria for classifying GGO or BAC are obscure, our classification using CT number histograms is objective and can be used to indicate not only BAC but also minimally invasive adenocarcinoma.

Although limited resection could be a reasonable approach for T1 N0 M0 lung carcinoma, it has been reported that lymph node metastases are found in about $20 \%$ of clinical T1 N0 M0 adenocarcinomas. ${ }^{10-14}$ In 1995, the Lung Cancer Study Group reported the results of a randomized control trial of a comparison between limited resection and lobectomy for clinical T1 N1 M0 non-small cell lung cancer. ${ }^{27}$ This trial demonstrated the inferiority of limited resection in terms of local relapse and prognosis because the patients should include pathological N1 or N2 disease. Besides, tumor involvement of the intratumoral vessels or pleura could also cause a local relapse after limited resection even for pathological NO disease due to spread of tumor cells into lymphatic vessels outside the primary tumor, for example, into intrapulmonary lymphatic tissue and subpleural lymphatic vessels. ${ }^{28} \mathrm{We}$ showed that adenocarcinomas with 1 peak at a low CT number had less frequent tumor involvement of the vessels or pleura than those with 1 peak at a high CT number and those with 2 peaks in pathological N0 disease.

We conclude that clinical T1 N0 M0 adenocarcinomas with 1 peak at a low CT number on histogram analysis usually do not metastasize to the lymph nodes or invade the intratumoral vessels or pleura. Therefore, limited surgical resection could be indicated for this type of adenocarcinoma, especially for elderly patients or patients with poor pulmonary function.

\section{References}

1. Kaneko M, Eguchi K, Ohmatsu H, et al. Peripheral lung cancer: screening and detection with low-dose spiral CT versus radiography. Radiology. 1996;201:798-802.

2. Yankelevitz DF, Gupta R, Zhao B, Henschke CI. Small pulmonary nodules: evaluation with repeat CT-preliminary experience. Radiology. 1999;212:561-6.

3. Henschke CI, Yankelevitz DF. CT screening for lung cancer. Radiol Clin North Am. 2000;38:487-95.

4. Nomori H, Horio H, Fuyuno G, Kobayashi R, Morinaga S, Suemasu K. Lung adenocarcinomas diagnosed by open or thoracoscopic vs. bronchoscopic biopsy. Chest. 1998;114:40-4.

5. Nomori $\mathrm{H}$, Horio $\mathrm{H}$. Colored collagen is a long-lasting point marker for small pulmonary nodules in thoracoscopic operations. Ann Thorac Surg. 1996;61:1070-3. 
6. Nomori H, Horio H, Naruke T, Suemasu K. Fluoroscopy-assisted thoracoscopic resection of lung nodules marked with lipiodol. Ann Thorac Surg. 2002;74:170-3.

7. Yoshikawa K, Tsubota N, Kodama K, Ayabe H, Taki T, Mori T. Prospective study of extended segmentectomy for small lung tumors. Ann Thorac Surg. 2002;73:1055-9.

8. Kodama K, Doi O, Higashiyama M, Yokouchi H. Intentional limited resection for selected patients with T1 N0 M0 non-small cell lung cancer. J Thorac Cardiovasc Surg. 1997;114:347-53.

9. Suzuki K, Asamura H, Kusumoto M, Kondo H, Tsuchiya R. Early peripheral lung cancer: prognostic significance of ground glass opacity on thin-section computed tomographic scan. Ann Thorac Surg. 2002; 74:1635-9.

10. Suzuki K, Nagai K, Yoshida J, Nishimura M, Nishizaki Y. Predictors of lymph node and intrapulmonary metastasis in clinical stage IA non-small cell lung carcinoma. Ann Thorac Surg. 2001;72:352-6.

11. Asamura H, Nakayama H, Kondo H, Tsuchiya R, Shimosato Y, Naruke T. Lymph node involvement, recurrence, and prognosis in resected small, peripheral, non-small cell lung carcinomas: are these carcinomas candidates for video-assisted lobectomy? J Thorac Cardiovasc Surg. 1996;111:1125-34.

12. Naruke T, Goya T, Tsuchiya R, Suemasu K. The importance of surgery of non-small cell carcinoma of lung with mediastinal lymph node metastasis. Ann Thorac Surg. 1998;46:603-10.

13. Naruke T, Tsuchiya R, Kondo H, Nakayama H, Asamura H. Lymph node sampling in lung cancer: how should it be done? Eur J Cardiothorac Surg. 1999;16(Suppl 1):S17-24.

14. Nomori H, Horio H, Naruke T, Orikasa H, Yamazaki K, Suemasu K. Use of technetium-99m tin colloid for sentinel lymph node identification in non-small cell lung cancer. J Thorac Cardiovasc Surg. 2002; 124:486-92.

15. Kuriyama K, Seto M, Kasugai T, et al. Ground-glass opacity on thin-section CT: value in differentiating subtypes of adenocarcinoma of the lung. Am J Roentgenol. 1999;173:465-9.

16. Matsuguma H, Yokoi K, Anraku M, et al. Proportion of ground-glass opacity on high-resolution computed tomography in clinical T1 N0 M0 adenocarcinoma of the lung: a predictor of lymph node metastasis. J Thorac Cardiovasc Surg. 2002;124:278-84.
17. Higashiyama M, Kodama K, Yokouchi H, et al. Prognostic value of bronchiolo-alveolar carcinoma component of small lung adenocarcinoma. Ann Thorac Surg. 1999;68:2069-73.

18. Aoki $\mathrm{T}$, Nakata $\mathrm{H}$, Watanabe $\mathrm{H}$, et al. Evolution of peripheral lung adenocarcinomas: CT findings correlated with histology and tumor doubling time. Am J Roentgenol. 2000;174:763-8.

19. Kodama K, Higashiyama M, Yokouchi H, et al. Prognostic value of ground-glass opacity found in small lung adenocarcinoma on highresolution CT scanning. Lung Cancer. 2001;33:17-25.

20. Travis WD, Colby TV, Corrin B, Shimosato Y, Brambilla E, in collaboration with Sobin LH and pathologists from 14 Countries. World Health Organization international histological classification of tumours. Histological typing of lung and pleural tumours. 3rd ed. Berlin: Springer-Verlag; 1999.

21. Brambilla E, Tarvis WD, Colby TV, Corrin B, Shimosato Y. The new World Health Organization classification of lung tumours. Eur Respir J. 2002;18:1059-68.

22. Sobin LH, Wittekind Ch, editors. UICC: TNM classification of malignant tumours. 6th ed. New York: John Wiley \& Sons; 2002.

23. Noguchi M, Morikawa A, Kawasaki M, et al. Small adenocarcinoma of the lung. Histologic characteristics and prognosis. Cancer. 1995; 75:2844-52.

24. Suzuki K, Yokose T, Yoshida J, et al. Prognostic significance of the size of central fibrosis in peripheral adenocarcinoma of the lung. Ann Thorac Surg. 2000;69:893-7.

25. Shimosato Y, Suzuki A, Hashimoto T, et al. Prognostic implications of fibrotic focus (scar) in small peripheral lung cancers. Am J Surg Pathol. 1980;4:365-73.

26. Nomori H, Hirohashi S, Noguchi M, Matsuno Y, Shimosato Y. Tumor cell heterogeneity and subpopulations with metastatic ability in differentiated adenocarcinoma of the lung. Chest. 1991;99:934-40.

27. Ginsberg RJ, Rubinstein LV. Randomized trial of lobectomy versus limited resection for T1 N0 non-small cell lung cancer. Lung Cancer Study Group. Ann Thorac Surg. 1995;60:615-22.

28. Ichinose Y, Yano T, Yokoyama H, Inoue T, Asoh H, Katsuda Y. The correlation between tumor size and lymphatic vessel invasion in resected peripheral stage I non-small cell lung cancer. A potential risk of limited resection. J Thorac Cardiovasc Surg. 1994;108:684-6. 